\title{
The Regulation of Ghrelin Contributes to the Physiological Basis of Obesity
}

\author{
Susan Westfall (McGill University) \\ News Reporter (HSI 2011-2012)
}

\section{A New Perspective on Obesity}

Obesity is generally regarded as a disease of poor lifestyle habits. Albeit true, humans have an innate predisposition to obesity depending on several physiological characteristics. This physiological predisposition is supported by epidemiological evidence describing the susceptibility of individuals to obesity despite lifestyle choices. Today, the obesity epidemic is being described as both a lifestyle disease and a metabolic disorder created by the poor regulation of hormones including the regulator of appetite, the desire to have food, fat deposition, and levels of ghrelin.

\section{The Regulation of Ghrelin}

Ghrelin is a 28-amino acid stomach-derived hormone discovered in 1999 by a group in Japan. ${ }^{1}$ Uniquely, ghrelin is the only hormone that responds to the amount of food in the digestive tract by increasing appetite, food intake and accumulation of body fat. If a negative energy balance occurs, such as during fasting, ghrelin secretion increases, leading to increased appetite and ability to store fat deposits. $^{2}$

\section{People with disrupted sleep schedules tend to gain weight, despite a normal caloric intake.}

Ghrelin is regulated beyond its response to food. Even without food intake, ghrelin is rhythmically secreted from the stomach in a circadian manner. ${ }^{3}$ Dr. Alfonso Abizaid, of Carleton University in Ottawa, investigated how ghrelin secretion is regulated by the circadian clock. Briefly, the circadian clock is the endogenous 24-hour molecular oscillator that integrates daily environmental signals such as the sleep-wake cycle, socialization habits, and feeding rhythms into a robust 24-hour cycling of molecular components. These fundamental oscillations synchronize physiological aspects in order to optimize the body's response to daily environmental changes. For example, it is known that people with disrupted sleep schedules tend to gain weight, despite normal caloric intake. This can be attributed to misaligned circadian rhythms created by differing sleep schedules. The perfect coordination of appetite, eating time and digestion is mediated through circadian oscillations of factors like ghrelin, cortisol and

\section{Ghrelin is the only hormone that responds to the amount of food in the digestive tract by increasing appetite, food intake and accumulation of body fat.}

insulin. When these axes become misaligned, metabolic diseases, including obesity, are likely to manifest.

Ghrelin is not directly associated with energy balance alone. Stressful stimuli alter the normal circadian ghrelin release by increasing the desire to reach for energy-rich 'comfort foods'. This desire to eat high-energy foods (concentrated in simple sugars and fats) comes from the release of ghrelin, stimulation of dopaminergic neurons and the reward pathways which associate comfort with these foods. ${ }^{2}$

The mechanism of ghrelin action involves both peripheral signalling and important central neuronal signaling that controls the addictive and desirous nature of food consumption. Ghrelin acts directly on its receptors in the hypothalamic region of the brain stimulating the release of orexigenic peptides like neuropeptide Y (NPY). NPY then directly stimulates the desire for food as well as a sense of predictive reward towards consumption of energyrich foods. This is the very same pathway that makes psychostimulatory drugs like amphetamine, cocaine and alcohol highly addictive. ${ }^{4}$

Ghrelin, like addictive drugs, releases dopamine into the ventral tegmental area of the brain increasing the anticipation of rewards and the insatiable desire to have increasing amounts of the stimulus. This addictive 
phenotype explains the addictive nature of eating that many people suffering from obesity describe. ${ }^{5}$ Interestingly, ghrelin might be the strongest modulator of dopamine release stimulated by feeding making interventions against its action not only beneficial for curbing obesity, but also the psychostimulatory effects of addictive drugs.

\section{Prospective Treatments}

The discovery of physiological factors like ghrelin in the regulation of obesity has given new perspectives into pharmaceutical treatments for obesity. Ghrelin is already being used to reduce appetite and fatty deposits and to control blood sugar. Conversely, ghrelin analogs are beings used to stimulate the appetite of persons suffering from wasting disorders or degenerative diseases. ${ }^{2}$

Interestingly, the treatment of circadian misalignment that would consequently stabilize ghrelin release is another treatment avenue for obesity in shift-workers. Melatonin is a chronobiological hormone that when given as a supplement to people working shift work helps stabilize their circadian rhythms. It has been found that melatonin also helps to stabilize ghrelin rhythms and consequently balance the energetic usage of food. Melatonin has already been shown to be beneficial in the treatment of irritable bowel syndrome, regulation of $\mathrm{GI}$ mobility and obesity. ${ }^{6}$

\section{Conclusion}

Obesity is a disease which not only reflects poor lifestyle choices, but is also a result of the misalignment of physiological factors, including hormones like ghrelin, that regulate energy balance. Genetics or physiology cannot be blamed for an obese state; however, genetics can impose a predisposition to obesity. This new angle of obesity research in the molecular condition provides novel and promising possibilities towards therapeutics that can help those suffering from obesity.

\section{Acknowledgments}

Special Thanks to Dr. Alfonso Abizaid for his contribution and insights on the above article.

\section{References}

1. Kojima M, Hosoda H, Date $\mathrm{Y}$, Nakazato M, Matsuo H, Kangawa K. Ghrelin is a growth-hormone-releasing acylated peptide from stomach. Nature. 1999. 402(6762):656-660.

2. Abizaid A. Ghrelin and Dopamine: New Insights on the Peripheral Regulation of Appetite. J. Neuroendocrin.2009. 21:787-793.

3. LeSauter J, Hoque N, Weintraub M, Pfaff DW, Silver R. Stomach ghrelinsecreting cells as food entrainable circadian clocks. Proc Natl Acad Sci USA. 2009. 106:13582-13587.

4. Abizaid A, Liu Z, Andres Z, Shanabrough M, Borok E, Elsworth J, et al. (2006) Ghrelin modulates the activity and synaptic input organization of midbrain dopamine neurons while promoting appetite. J. Clin. Inv. 2006. 116(12):3229-3240

5. Kind S, Isaacs A, O'Farrell E, Abizaid A. Motivation to obtain preferred foods is enhanced by ghrelin in the ventral tegmental area. Hormones and Behav 2001. 60:572-580

6. Konturek PC, Brzozowski T, Konturek SJ. The Gut Clock. J. Physiol. Pharm. 2011. 62(2):139-150.

\section{Susan Westfall}

Susan's has recently completed her Master's Degree in the Integrated Program of Neuroscience from McGill Univerisity, Montreal. Her research focused on the interplay between the immune system and circadian regulation, the endogenous $24 \mathrm{~h}$ physiological rhythms. In addition to her interest in science, Susan recognizes the significance of alternative healing methods. Her focus now after the completion of her Master's, is to prove using the scientific dogma, that alternative health practices are reliable and should be incorporated into the western paradigm of health. 